\title{
Presssure-Tuning of the Photomagnetic Response of Heterostructured CoFe@CrCr-PBA Core@Shell Nanoparticles
}

\author{
Marcus K. Peprah ${ }^{\mathrm{a}}$, Derrick VanGennep ${ }^{\mathrm{a}}$, Pedro A. Quintero ${ }^{\mathrm{a}}$, Oliva N. Risset ${ }^{\mathrm{b}}$, Tatiana V. Brinzari ${ }^{\mathrm{a}}$, Carissa H. Li ${ }^{\mathrm{b}}$, \\ Matthieu F. Dumont ${ }^{\mathrm{a}}$, Jian-sheng Xia ${ }^{\mathrm{a}}$, James J. Hamlin ${ }^{\mathrm{a}}$, Daniel R. Talham ${ }^{\mathrm{b}, *}$, Mark W. Meisel ${ }^{\mathrm{a}, *}$ \\ ${ }^{a}$ Department of Physics and National High Magnetic Field Laboratory, University of Florida, Gainesville, FL 32611-8440, USA \\ ${ }^{b}$ Department of Chemistry, University of Florida, Gainesville, FL 32611-7200, USA
}

\begin{abstract}
Using a home-made, anvil pressure-cell mounted to a probe suitable for use with a commercial magnetometer, the photo and thermal responses of the magnetism of CoFe@CrCr-PBA (PBA = Prussian blue analogue) core@ shell nanoparticles were studied down to $5 \mathrm{~K}$ and up to $0.5 \mathrm{GPa}$ in $100 \mathrm{G}$. The effect of pressure on the magnetic ordering temperatures of the CoFe-PBA core $(\approx 25 \mathrm{~K})$ and the CrCr-PBA shell $(\approx 200 \mathrm{~K})$, along with a shift of the relaxation temperature of the photo-CTIST (charge-transfer-induced spin-transition) of the CoFe-PBA core $(\approx 125 \mathrm{~K})$, were similar to the behaviors reported for the single-phase materials. Specifically, although the magnetic ordering temperature of the CrCr-PBA shell shifted to higher temperatures, the relaxation temperature of the photo-CTIST of the CoFe-PBA core moved to lower temperatures when the pressure was increased, thereby lowering the temperature range under which the CrCr-PBA component can be photoswitched.
\end{abstract}

Keywords: core-shell nanoparticles, heterostructures, Prussian blue analogues, photomagnetism

\section{Introduction}

By their nature, molecule-based magnetic systems are significantly "softer" than their solid-state counterparts. Consequently, external pressure and strain are being widely applied to coordination polymers and single-molecule magnets to probe a range of emerging multiferroic behavior [1-7]. An important coordination polymer family are the Prussian blue analogues, which are cubic cyanide-bridged mixed-metal networks of general formula $\mathrm{A}_{j} \mathrm{M}_{k}\left[\mathrm{M}^{\prime}(\mathrm{CN})_{6}\right]_{l} \cdot n \mathrm{H}_{2} \mathrm{O}$, where the monovalent ion $\mathrm{A}$ is located in the intersticies, as needed, to balance charge. The shorthand $\mathrm{MM}^{\prime}$-PBA can be used to indicate an analogue of two first-row transition metals, $\mathrm{M}$ and $\mathrm{M}^{\prime}$. Recently, photocontrol of the magnetic response in $\mathrm{CoFe} @ \mathrm{CrCr}-$ PBA core@shell nanoparticles has been demonstrated to persist to $125 \mathrm{~K}$ [8]. In this system, the persistent, photoinduced changes of magnetism of the heterostructure are compromised by the thermal relaxation of the structural changes associated with the photo-CTIST (charge-transfer-induced spin-transition) effect of the CoFe-PBA core, since the light-induced phase transition of the core couples to the shell whose magnetic response is modified.

This investigation of $\mathrm{CoFe} @ \mathrm{CrCr}$-PBA core@ shell nanoparticles evolved from studies of $\mathrm{CoFe} @ \mathrm{NiCr}-\mathrm{PBA}$ hetrostructures (thin films and nanoparticles) in which the photocontrol of the magnetic response of the NiCr-PBA component was observed up to its ordering temperature, $T_{c}=70 \mathrm{~K}[9,10]$, as the lightinduced state of the CoFe-PBA core persists to even higher

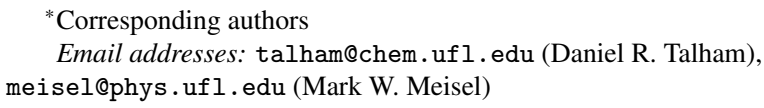

temperature. In contrast, although photocontrol of the magnetic response of CoFe-PBA@CrCr-PBA is observed to higher temperature, up to $125 \mathrm{~K}$, which is the relaxation temperature of the metastable light-induced state of the core, the magnetic changes do not persist up to $T_{c}=200 \mathrm{~K}$, the ferrimagnetic transition temperature of the CrCr-PBA shell. The motivation for the present study was to see if the photoinduced magnetic changes could be shifted by external pressure from $125 \mathrm{~K}$ to 200 K. Specifically, a home-made, anvil pressure-cell suitable for use with a commercial magnetometer was used to investigate the photo and thermal responses of the low-field magnetic response of $\mathrm{CoFe} @ \mathrm{CrCr}$-PBA core@ shell nanoparticles, whose ambient pressure response has been reported elsewhere [8].

Using PBA heterostructures, a series of comprehensive studies by several groups have elucidated the robust magnetomechanical coupling at the interface of the two components and the interplay between the size of the cores and the thicknesses of the shells for observing photoinduced changes in the magnetic response [11-14]. Although the effect of external pressure on the magnetic behavior of several PBAs has been reported, including CoFe-PBA [15-18] and CrCr-PBA [19] that are the components of the system used in present study, the magnetic changes induced by light and pressure have not been previously reported for PBA heterostructures. Herein, the observed effects of pressure on the magnetic ordering temperatures of the CoFe-PBA core $\left(T_{\mathrm{N}, \mathrm{c}} \approx 25 \mathrm{~K}\right)^{1}$ and the CrCr-PBA shell $\left(T_{\mathrm{N}, \mathrm{s}} \approx 200 \mathrm{~K}\right)$, along with the shift of the temperature where

\footnotetext{
${ }^{1}$ The Néel temperatures of the PBA constituents of the core and shell are designated as $T_{\mathrm{N}, \mathrm{c}}$ and $T_{\mathrm{N}, \mathrm{s}}$, respectively.
} 
the photo-CTIST of the CoFe-PBA core relaxes $\left(T_{\mathrm{R}} \approx 125 \mathrm{~K}\right)$, are similar to the behaviors reported for the single-phase materials $[15,16,18,19]$. In other words, although the $T_{\mathrm{N}, \mathrm{s}}$ of the CrCr-PBA shell shifted to higher temperatures, the $T_{\mathrm{R}}$ of the $\mathrm{CoFe}-\mathrm{PBA}$ core moved to lower temperatures when the pressure was increased. These results provide a guide for future approaches seeking to observe photocontrolled magnetic response at higher temperatures in these kinds of heterostructures, and chemical tuning of the CoFe constituent will play a significant role $[17,20,21]$.

\section{Experimental Methods}

\subsection{Sample synthesis and characterization}

The core@ shell nanoparticles of $\left\{\mathrm{Rb}_{0.2} \mathrm{Co}\left[\mathrm{Fe}(\mathrm{CN})_{6}\right]_{0.7}\right\}_{0.8} @$ $\left\{\mathrm{K}_{0.1} \mathrm{Cr}\left[\mathrm{Cr}(\mathrm{CN})_{6}\right]_{0.7}\right\}_{0.2} \cdot 4 \mathrm{H}_{2} \mathrm{O}(\mathrm{CoFe} @ \mathrm{CrCr}-\mathrm{PBA})$ used for this study were from the same batch of material whose synthesis protocols and structural properties were described elsewhere $[8,22]$. The cubic cores of CoFe-PBA have sides of $172 \pm 13 \mathrm{~nm}$, whereas the CrCr-PBA shell has a thickness of $9 \pm 2 \mathrm{~nm}$. Attempts to grow thicker CrCr-PBA shells on the $\mathrm{CoFe}-\mathrm{PBA}$ core seed particles resulted in the nucleation of pure CrCr-PBA particles, so the present sample represents the thickest $\mathrm{CrCr}$-PBA shell grown to date. The sample used in this pressure study had a mass of approximately $4.0 \mu \mathrm{g}$.

\subsection{Anvil pressure cell}

The homemade optical-access pressure cell for use with a commerical magnetometer follows closely the miniature anvil cell described by Giriat et al. [23] and is based on the Tozer turnbuckle design [24]. A complete set of schematics and detailed drawings are available elsewhere and online [18]. A CuTi alloy was used as the primary component of the cell because of its low magnetization properties. The main cell body consists of an octagonal prism that measures $7 \mathrm{~mm}$ in length and $7 \mathrm{~mm}$ in diameter. Four $1 \mathrm{~mm}$ diameter holes are placed on four flat sides of the cell body and serve as viewing windows for the culets and the gasket. In addition, the cell consists of two pressurization screws (6 $\mathrm{mm}$ in diameter and $3 \mathrm{~mm}$ in length) that are counter threaded to provide the the turnbuckle mechanism. Atop the screws, three machined holes form a triangle, and a fourth hole $(1 \mathrm{~mm})$ at the center provides access for an optical fiber when irradiating the sample space. The application of pressure is delivered by silicon carbide ( $\mathrm{SiC}$ ) anvils, whose culet diameter was $800 \mu \mathrm{m}$. A BeCu gasket with a $290 \mu \mathrm{m}$ hole confined the sample, a small Ruby $\left(\mathrm{Al}_{2} \mathrm{O}_{3}\right.$ with $\mathrm{Cr}^{3+}$ doping) whose fluorescence served as the manometer by following established protocols $[25,26]$, and a tiny amount Daphne-7373 oil employed as the pressure transmitting fluid [27-31]. The fluorescence spectra were acquired by a home-made, inexpensive mini-spectrometer [32], which provided excitation at $405 \mathrm{~nm}$. The resulting emission at $694.3 \mathrm{~nm}$ was used to provide pressure measurements at all temperatures $(2 \mathrm{~K} \leq T \leq 300 \mathrm{~K})$. During the final assembly stages an optical microscope was used to position the anvils, and a defect was noticed at the edge of one of the anvils. This defect restricted the present work to pressures $P \leq 0.5 \mathrm{GPa}$, while the minimum pressure value, typically $P \approx 0.05 \mathrm{GPa}$, is needed to insure a snug (leak-tight) fit of the anvils to the gasket. It is noteworthy that a pressure of $0.5 \mathrm{GPa}$ has a significant effect on the magnetic and photomagnetic response of single-component CoFe-PBA [15, 16, 18], and examples of this response [18] are provided in the Supplementary Material (SM), which is available online. In addition, the temperature dependence of the magnetic signal arising from the cell sans sample is provided in the SM.

\subsection{Protocols for measuring the magnetic response}

To avoid quenching the thermal-CTIST transition of the CoFe-PBA component [33] and straining the single optical fiber (with a $600 \mu \mathrm{m}$ diameter active optical core and an overall outer diameter of $720 \mu \mathrm{m}$ ) extending from room temperature down to the sample cell, the cooling rates were restricted to $\leq 3 \mathrm{~K} / \mathrm{min}$ down to $100 \mathrm{~K}$ and then to $\leq 5 \mathrm{~K} / \mathrm{min}$ at lower temperatures. Similarly, warming rates were limited to $\leq 2 \mathrm{~K} / \mathrm{min}$ for $T \leq 10 \mathrm{~K}$ and $5 \mathrm{~K} / \mathrm{min}$ for $10 \mathrm{~K}<T \leq 300 \mathrm{~K}$. The cooling/warming/measuring processes were always in the presence of a static, applied magnetic field of $100 \mathrm{G}$.

The dark state data were acquired with a black cover on the room temperature end of the optical fiber. The light state was achieved at $5 \mathrm{~K}$ by irradiating the sample with unfiltered light generated by a halogen bulb after waiting for nominally 1 hour before starting the irradiation process, which typically lasted about 2 hours. When the light was switched off and the fiber was covered, the sample remained at $5 \mathrm{~K}$ for another hour before the warming process was initiated. Typical plots of the magnetic response as a function of time are shown in the SM section.

\section{Results and Data Analysis}

The temperature dependences of the magnetic susceptibility $\chi=M / B$, where $M$ is the magnetization and $B=100 \mathrm{G}$, are shown for the dark and light states at pressures $P=0.05 \mathrm{GPa}$ (essentially ambient pressure) and $0.5 \mathrm{GPa}$, Fig. 1. The background signal from the pressure cell has not been subtracted. An effective means to judge the photoinduced changes is to plot the differences between the light and dark states, namely $\Delta \chi=\chi($ Light $)-\chi($ Dark $)$. This type of analysis allows for a simple and efficient method of eliminating the background signals arising from the pressure cell as these magnetic signals do not possess a photoactive response. In addition, the changes at low temperature, $T \lesssim 25 \mathrm{~K}$, dominate the subtle changes at higher temperatures, $25 \mathrm{~K} \lesssim T<300 \mathrm{~K}$, so these regimes are plotted separately in Figs. 2 and 3.

Although the $\Delta \chi$ data sets are weak and somewhat noisy for $T \gtrsim 25 \mathrm{~K}$, Fig. 3(a), it is important to note these changes in the magnetic response of the core@shell particle arise from the distortion of the magnetic domains of the $\mathrm{CrCr}$-PBA shell in response to structural changes associated with the lightinduced CTIST in the CoFe-PBA core. Furthermore, the hightemperature response of the system is detectable because of the 


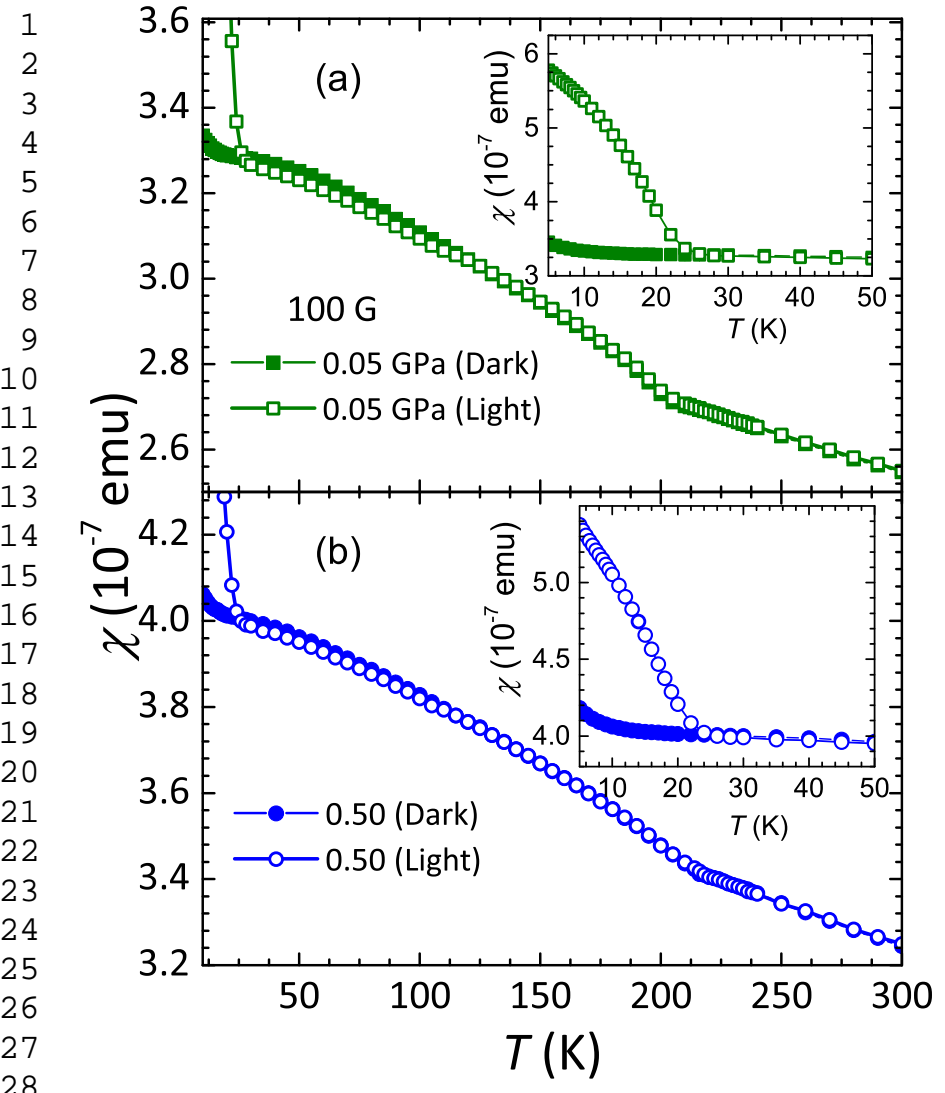

29

30

31

32

The results presented in the preceding section for CoFe@CrCr-PBA nano-sized heterostructures can be discussed

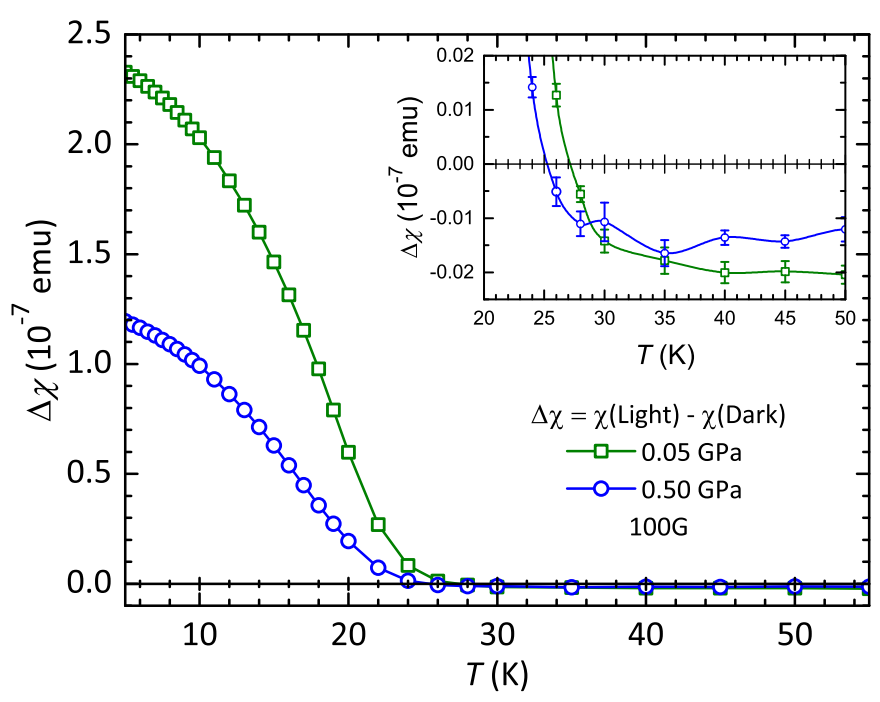

Figure 2: The low temperature, $T \leq 55 \mathrm{~K}$, data from Fig. 1 are shown as the differences between the light and dark states. The application of pressure causes an overall decrease in the photoinduced magnetic changes for $T \lesssim 25 \mathrm{~K}$. These changes arise from the CoFe-PBA core and are consistent with the responses observed with single-component CoFe-PBA as discussed in the text. The inset provides an expanded view of the region where the differences become negative. Lines are guides for the eyes.

in the context of an evolving interplay between the electronic configurations of the transition metal ions as tuned by temperature, irradiation, and pressure. It is important to note that chemical tuning of the magnetic response is held constant in this work, thereby allowing an investigation of the other three variables. During the systematic discussion of the various phases of the magnetism under this diverse set of conditions and histories, some jargon will be used so it is perhaps helpful to review this terminology in advance of the detailed analysis. Specifically, $H S$ and $L S$ will refer to high-spin and low-spin states, respectively, and the CTIST (charge-transfer-induced spin-transition) effect can be induced by either thermal (at $\mathrm{T}_{\mathrm{CTIST}}$ ) or photo processes, where the relaxation of the photo-CTIST occurs at a temperature $T_{\mathrm{R}}$. Irradiation of the dark state establishes the light state in the core by a photo-CTIST process, and this phenomenon is known as photoinduced magnetism (PIM) below the magnetic ordering temperature of the CoFe-PBA near $24 \mathrm{~K}$ $[34,35]$. At higher temperatures, the light state persists as the CoFe spin pairs remain in their paramagnetic $(S \neq 0)$ states until thermally relaxed near $125 \mathrm{~K}$. Finally, the pressure-induced electron-transfer (PIET) effect causes the low volume $(S=0)$ non-magnetic states of CoFe-PBA to be increased [15-18], as pressure favors the low volume state causing an increase in the temperature range over which it is established. Of course, it is widely known that external pressure can alter bond distances and angles thereby resulting in modificaitons of the magnetic exchange interactions. For ferrimagnets, increasing pressure ususally leads to increased overlap of the magnetic orbitals, thereby increasing the antiferromagnetic interactions and generating a higher transition temperature. Contrastingly in ferromagnets, increasing pressure typically strengthens the competing antiferromagnetic interactions, results in a decrease of 


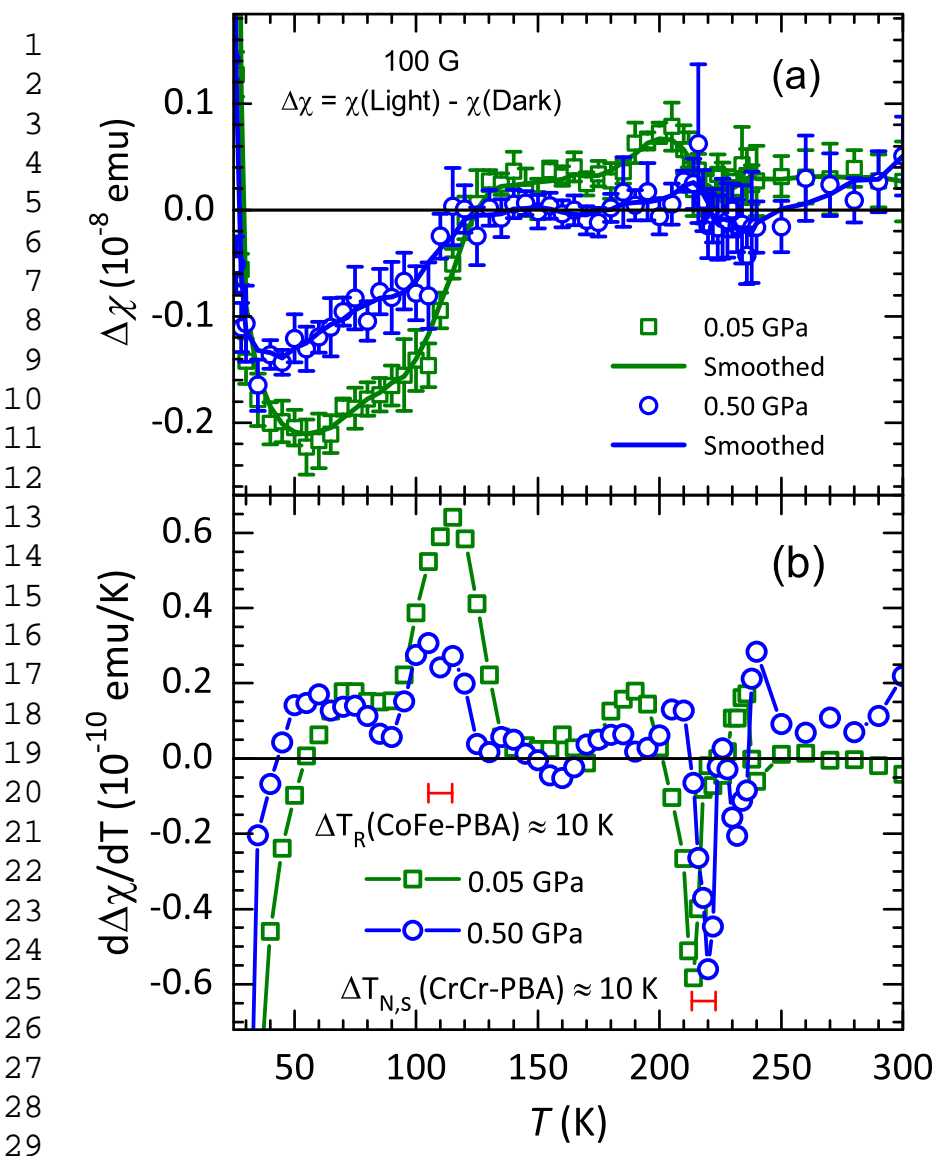

30

31 tometry results on other core@ shell systems with CoFe-cores and different PBA shells [13, 14, 39].

At $5 \mathrm{~K}$, irradiation generates the light state, and a large number of metastable $\mathrm{Fe}^{3+}(L S, S=1 / 2)-\mathrm{CN}-\mathrm{Co}^{2+}(H S, S=3 / 2)$ pairs are generated and persist after the light is extinguished. The photo-CTIST establishs the PIM state in the CoFe-PBA core, and the thermal-CTIST strain, which formed while cooling, is relaxed as many of the CoFe pairs assume lattice dimensions close to those of the room temperature state [12]. The magnetic domains in the CrCr-PBA shells now experience a different magneto-crystalline anisotropy as long as the applied magnetic field is below nominally a few kG [18, 40]. Furthermore, this new metastable magnetomechanical arrangement of the ferrimagnetic domains is maintained until the photo-CTIST is relaxed at $T_{\mathrm{R}} \approx 125 \mathrm{~K}$.

Given this description of where thermal and photo effects are varied while the chemical composition and low magnetic field are held constant, pressure can be added as another external variable. Although pressure studies of single-phase PBAs have appeared in the literature, this study is the first using PBA heterostructures while also maintaining irradiation as a variable. From this viewpoint, it is important to stress that the external pressure was always applied at room temperature. On the other hand, three other strain-related processes occur at lower temperatures, and these mechanisms are the effective thermalstrain induced by lattice mismatch while slow-cooling through the thermal-CTIST transition of the CoFe-PBA core, the subsequent metastable release of some of this effective thermal-strain via the photo-CTIST transition by irradiation, and the final relaxation of this effective thermal-strain at $T_{\mathrm{R}}$, where the lattices of the core and shell resume their equilibrium configurations.

Application of pressure at room temperature drives magnetic $(S \neq 0)$ CoFe pairs to become locked in their nonmagentic $(S=0)$ states because the reduction of the volume favors the $S=0$ configuration. Consequently with an increase of external pressure at room temperature, fewer $\mathrm{CoFe}$ pairs can be photoswitched at low temperatures from $S=0$ to $S \neq 0$ arrangements, so the magnetic signal of the light state decreases with increased pressure applied at room temperature. This trend is manifested in the data shown in Fig. 2, where the number of spins experiencing PIM are reduced with increased pressure applied at room temperature. Another way to describe this volume reduction (by either externally applied pressure or by chemical tuning) is to recognize that $T_{\mathrm{CTIST}}$ increases while $T_{\mathrm{R}}$ correspondingly decreases with increasing external or chemical pressure [20, 21]. Indeed, this reduction of $T_{\mathrm{R}}$ is detected, Fig. 3(b). Contrastingly for the $\mathrm{CrCr}$-PBA shell, $T_{\mathrm{N}, \mathrm{s}}$ increases in a manner that is consistent with studies on the pure-phase [19].

\section{Conclusions}

In summary, the pressure dependences of the magnetic responses of the core and shell components of CoFe@CrCr-PBA nanoparticles are the same as those observed in the single-phase constituents. In other words, the application of pressure favors the nonmagnetic configurations in the CoFe-PBA core, and as a 
result, the relaxation of the photo-CTIST moves to lower temperatures. This trend is opposite of the one needed to increase $T_{\mathrm{R}}$ of the core to be greater than or similar to the magnetic transition temperature of the shell. On the other hand, the application of pressure does increase this magnetic transition temperature, at least for the CrCr-PBA shell used in this work. Ultimately, the next step to realizing persistent photocontrolled magnetic switching at higher temperatures may be to tune the chemical composition [17] or to explore options afforded by spin-crossover complexes [40-44].

\section{Acknowledgments}

This work was supported by the NSF via DMR1202033 (MWM), DMR-1405439 (DRT), and DMR-1157490 (NHMFL), and by the State of Florida. Instrumentation for the pressure cell was funded by the NHMFL User Collaboration Grants Program (JJH). We gratefully acknowledge Y. Takano, who made the CuTi-alloy available, S. L. Cooper, who provided details about $\mathrm{SiC}$ anvils, and J. M. Cain, who offered some enlightening comments.

[1] P. Parois, S. A. Moggach, J. Sanchez-Benitez, K. V. Kamenev, A. R. Lennie, J. E. Warren, E. K. Brechin, S. Parsons, M. Murrie, Pressureinduced Jahn-Teller switching in a $\mathrm{Mn}_{12}$ nanomagnet, Chemical Communications 46 (2010) 1881-1883, doi:10.1039/B923962F.

[2] A. Prescimone, C. Morien, D. Allan, J. A. Schlueter, S. W. Tozer, J. L. Manson, S. Parsons, E. K. Brechin, S. Hill, Pressure-Driven Orbital Reorientations and Coordination-Sphere Reconstructions in $\left[\mathrm{CuF}_{2}\left(\mathrm{H}_{2} \mathrm{O}\right)_{2}(\mathrm{pyz})\right]$, Angewandte Chemie International Edition 51 (30) (2012) 7490-7494, doi:10.1002/anie.201202367.

[3] K. R. O'Neal, T. V. Brinzari, J. B. Wright, C. Ma, S. Giri, J. A. Schlueter, Q. Wang, P. Jena, Z. Liu, J. L. Musfeldt, Pressure-Induced Magnetic Crossover Driven by Hydrogen Bonding in $\mathrm{CuF}_{2}\left(\mathrm{H}_{2} \mathrm{O}\right)_{2}$ (3-chloropyridine), Scientific Reports 4 (2014) 6054, doi: $10.1038 /$ srep06054.

[4] P. A. Quintero, D. Rajan, M. K. Peprah, T. V. Brinzari, R. S. Fishman, D. R. Talham, M. W. Meisel, Pressure-induced enhancement of the magnetic anisotropy in $\mathrm{Mn}\left(\mathrm{N}(\mathrm{CN})_{2}\right)_{2}$, Physical Review B 91 (2015) 014439, doi:10.1103/PhysRevB.91.014439.

[5] D. Pinkowicz, M. Rams, M. Mišek, K. V. Kamenev, H. Tomkowiak, A. Katrusiak, B. Sieklucka, Enforcing Multifunctionality: A PressureInduced Spin-Crossover Photomagnet, Journal of the American Chemical Society 137 (27) (2015) 8795-8802, doi:10.1021/jacs.5b04303.

[6] S. A. Corrales, J. M. Cain, K. A. Uhlig, A. M. Mowson, C. Papatriantafyllopoulou, M. K. Peprah, A. Ozarowski, A. J. Tasiopoulos, G. Christou, M. W. Meisel, C. Lampropoulos, Introducing Dimensionality to the Archetypical $\mathrm{Mn}_{12}$ Single-Molecule Magnet: a Family of $\left[\mathrm{Mn}_{12}\right]_{n}$ Chains, Inorganic Chemistry 55 (4) (2016) 1367-1369, doi: 10.1021/acs.inorgchem.6b00058.

[7] K. R. O'Neal, J. Zhou, J. G. Cherian, M. M. Turnbull, C. P. Landee, P. Jena, Z. Liu, J. L. Musfeldt, Pressure-induced structural transition in copper pyrazine dinitrate and implications for quantum magnetism, Physical Review B 93 (2016) 104409, doi:10.1103/PhysRevB.93.104409.

[8] O. N. Risset, T. V. Brinzari, M. W. Meisel, D. R. Talham, Light Switchable Magnetism in a Coordination Polymer Heterostructure Combining the Magnetic Potassium Chromiumhexacyanochromate with the Light-Responsive Rubidium Cobalthexacyanoferrate, Chemistry of Materials 27 (18) (2015) 6185-6188, doi:10.1021/acs.chemmater.5b02785.

[9] D. M. Pajerowski, M. J. Andrus, J. E. Gardner, E. S. Knowles, M. W. Meisel, D. R. Talham, Persistent Photoinduced Magnetism in Heterostructures of Prussian Blue Analogues, Journal of the American Chemical Society 132 (12) (2010) 4058-4059, doi:10.1021/ja100246n.

[10] M. F. Dumont, E. S. Knowles, A. Guiet, D. M. Pajerowski, A. Gomez, S. W. Kycia, M. W. Meisel, D. R. Talham, Photoin- duced Magnetism in Core/Shell Prussian Blue Analogue Heterostructures of $\mathrm{K}_{j} \mathrm{Ni}_{k}\left[\mathrm{Cr}(\mathrm{CN})_{6}\right]_{l} \cdot n \mathrm{H}_{2} \mathrm{O}$ with $\mathrm{Rb}_{a} \mathrm{Co}_{b}\left[\mathrm{Fe}(\mathrm{CN})_{6}\right]_{c} \cdot m \mathrm{H}_{2} \mathrm{O}$, Inorganic Chemistry 50 (10) (2011) 4295-4300, doi:10.1021/ic1022054.

[11] M. Presle, I. Maurin, F. Maroun, R. Corts, L. Lu, R. S. Hassan, E. Larquet, J.-M. Guigner, E. Rivire, J. P. Wright, J.-P. Boilot, T. Gacoin, Photostrictive/Piezomagnetic CoreShell Particles Based on Prussian Blue Analogues: Evidence for Confinement Effects?, The Journal of Physical Chemistry C 118 (24) (2014) 13186-13195, doi:10.1021/jp501758b.

[12] D. M. Pajerowski, B. Ravel, C. H. Li, M. F. Dumont, D. R. Talham, X-ray Absorption Study of Structural Coupling in Photomagnetic Prussian Blue Analogue Core@Shell Particles, Chemistry of Materials 26 (8) (2014) 2586-2594, doi:10.1021/cm4042007.

[13] O. N. Risset, P. A. Quintero, T. V. Brinzari, M. J. Andrus, M. W. Lufaso, M. W. Meisel, D. R. Talham, Light-Induced Changes in Magnetism in a Coordination Polymer Heterostructure, $\mathrm{Rb}_{0.24} \mathrm{Co}\left[\mathrm{Fe}(\mathrm{CN})_{6}\right]_{0.74} @ \mathrm{~K}_{0.10} \mathrm{Co}\left[\mathrm{Cr}(\mathrm{CN})_{6}\right]_{0.70} \cdot n \mathrm{H}_{2} \mathrm{O}$ and the Role of the Shell Thickness on the Properties of Both Core and Shell, Journal of the American Chemical Society 136 (44) (2014) 15660-15669, doi: 10.1021/ja5084283.

[14] A. C. Felts, M. J. Andrus, E. S. Knowles, P. A. Quintero, A. R. Ahir, O. N. Risset, C. H. Li, I. Maurin, G. J. Halder, K. A. Abboud, M. W. Meisel, D. R. Talham, Evidence for Interface-Induced Strain and Its Influence on Photomagnetism in Prussian Blue Analogue CoreShell Heterostructures, $\mathrm{Rb}_{a} \mathrm{Co}_{b}\left[\mathrm{Fe}(\mathrm{CN})_{6}\right]_{c} \cdot m \mathrm{H}_{2} \mathrm{O} @ \mathrm{~K}_{j} \mathrm{Ni}_{k}\left[\mathrm{Cr}(\mathrm{CN})_{6}\right]_{l} \cdot n \mathrm{H}_{2} \mathrm{O}$, The Journal of Physical Chemistry C 120 (10) (2016) 5420-5429, doi: 10.1021/acs.jpcc.5b10761.

[15] V. Ksenofontov, G. Levchenko, S. Reiman, P. Gütlich, A. Bleuzen, V. Escax, M. Verdaguer, Pressure-induced electron transfer in ferrimagnetic Prussian blue analogs, Physical Review B 68 (2003) 024415, doi: 10.1103/PhysRevB.68.024415.

[16] V. Ksenofontov, A. B. Gaspar, P. Gütlich, Spin Crossover in Transition Metal Compounds III, chap. Pressure Effect Studies on Spin Crossover and Valence Tautomeric Systems, Springer Berlin Heidelberg, Berlin, Heidelberg, ISBN 978-3-540-44984-3, 23-64, doi: 10.1007/b95421, 2004.

[17] J.-D. Cafun, J. Lejeune, F. Baudelet, P. Dumas, J.-P. Itié, A. Bleuzen, Room-Temperature Photoinduced Electron Transfer in a Prussian Blue Analogue under Hydrostatic Pressure, Angewandte Chemie International Edition 51 (36) (2012) 9146-9148, doi:10.1002/anie.201203943.

[18] M. K. A. Peprah, Influence of Pressure and Light on the Magnetic Properties of Prussian Blue Analogues and HofmannLike Frameworks, Ph.D. thesis, University of Florida, URL http://ufdc.ufl.edu/UFE0049032/00001, 2015.

[19] Z. Mitróvá, S. Mat'aš, M. Zentková, Z. Arnold, J. Kamarád, Effect of Pressure on Magnetic Properties of Hexacyanochromates, Acta Physica Polonica A 113 (2008) 469 - 472, doi:10.12693/APhysPolA.113.469.

[20] V. Escax, A. Bleuzen, C. C. dit Moulin, F. Villain, A. Goujon, F. Varret, M. Verdaguer, Photoinduced Ferrimagnetic Systems in Prussian Blue Analogues $\mathrm{CI}_{x} \mathrm{Co}_{4}\left[\mathrm{Fe}(\mathrm{CN})_{6}\right]_{y}(\mathrm{CI}=$ Alkali Cation). 3. Control of the Photo- and Thermally Induced Electron Transfer by the $\left[\mathrm{Fe}(\mathrm{CN})_{6}\right] \mathrm{Va}-$ cancies in Cesium Derivatives, Journal of the American Chemical Society 123 (50) (2001) 12536-12543, doi:10.1021/ja011296r.

[21] N. Shimamoto, S. ichi Ohkoshi, O. Sato, K. Hashimoto, Control of Charge-Transfer-Induced Spin Transition Temperature on CobaltIron Prussian Blue Analogues, Inorganic Chemistry 41 (4) (2002) 678-684, doi:10.1021/ic010915u.

[22] O. N. Risset, Magnetic and Electrical-Transport Studies of Molecule-Based Magnets, Ph.D. thesis, University of Florida, URL http://ufdc.ufl.edu/UFE0047327/00001, 2015.

[23] G. Giriat, W. Wang, J. P. Attfield, A. D. Huxley, K. V. Kamenev, Turnbuckle diamond anvil cell for high-pressure measurements in a superconducting quantum interference device magnetometer, Review of Scientific Instruments 81 (7) 073905, doi:10.1063/1.3465311.

[24] D. E. Graf, R. L. Stillwell, K. M. Purcell, S. W. Tozer, Nonmetallic gasket and miniature plastic turnbuckle diamond anvil cell for pulsed magnetic field studies at cryogenic temperatures, High Pressure Research 31 (4) (2011) 533-543, doi:10.1080/08957959.2011.633909.

[25] A. D. Chijioke, W. J. Nellis, A. Soldatov, I. F. Silvera, The ruby pressure standard to 150GPa, Journal of Applied Physics 98 (11) 114905, doi: 10.1063/1.2135877.

[26] S. Buchsbaum, R. L. Mills, D. Schiferl, Phase diagram of nitrogen de- 
termined by Raman spectroscopy from 15 to $300 \mathrm{~K}$ at pressures to 52 GPa, The Journal of Physical Chemistry 88 (12) (1984) 2522-2525, doi: 10.1021/j150656a018.

[27] K. Kamishima, M. Hagiwara, H. Yoshida, Investigation of a strong titanium alloy KS15-5-3 and the application to a high pressure apparatus for magnetization measurements, Review of Scientific Instruments 72 (2) (2001) 1472-1476, doi:10.1063/1.1337074.

[28] J. Kamarád, Z. Machátová, Z. Arnold, High pressure cells for magnetic measurementsDestruction and functional tests, Review of Scientific Instruments 75 (11) (2004) 5022-5025, doi:10.1063/1.1808122.

[29] Y. Uwatoko, T. Fujiwara, M. Hedo, F. Tomioka, I. Umehara, The development of a high pressure micro-cell for magnetization and specific heat measurements: the effect of pressure on the magnetism in CeAg, Journal of Physics: Condensed Matter 17 (11) (2005) S1011, doi:10.1088/09538984/17/11/033.

[30] K. Yokogawa, K. Murata, H. Yoshino, S. Aoyama, Solidification of HighPressure Medium Daphne 7373, Japanese Journal of Applied Physics 46 (6R) (2007) 3636, doi:10.1143/JJAP.46.3636.

[31] M. S. Torikachvili, S. K. Kim, E. Colombier, S. L. Bud'ko, P. C. Canfield, Solidification and loss of hydrostaticity in liquid media used for pressure measurements, Review of Scientific Instruments 86 (12) 123904, doi: $10.1063 / 1.4937478$

[32] J. J. Hamlin, et al., to be published, 2016.

[33] J.-H. Park, F. Frye, N. Anderson, D. Pajerowski, Y. Huh, D. Talham, M. Meisel, Cooling rate-dependent charge-transfer-induced spin transition in $\mathrm{K}_{0.4} \mathrm{Co}_{1.3}\left[\mathrm{Fe}(\mathrm{CN})_{6}\right] \cdot n \mathrm{H}_{2} \mathrm{O}$ Prussian blue analog, Journal of Magnetism and Magnetic Materials 310 (2, Part 2) (2007) 1458 - 1459, doi: 10.1016/j.jmmm.2006.10.468.

[34] M. Verdaguer, Molecular Electronics Emerges from Molecular Magnetism, Science 272 (5262) (1996) 698-699, doi: 10.1126/science.272.5262.698.

[35] O. Sato, T. Iyoda, A. Fujishima, K. Hashimoto, Photoinduced Magnetization of a Cobalt-Iron Cyanide, Science 272 (5262) (1996) 704-705, doi:10.1126/science.272.5262.704.

[36] C.-H. Lee, C.-M. Wu, E. Batsaikhan, H.-C. Li, C. H. Li, M. K. Peprah, D. R. Talham, M. W. Meisel, W.-H. Li, Complex Magnetic Phases in Nanosized Core@Shell Prussian Blue Analogue Cubes: $\quad \mathrm{Rb}_{0.48} \mathrm{Co}\left[\mathrm{Fe}(\mathrm{CN})_{6}\right]_{0.75}\left[\left(\mathrm{H}_{2} \mathrm{O}\right)_{6}\right]_{0.25} \cdot 0.34 \mathrm{H}_{2} \mathrm{O} @$ $\mathrm{K}_{0.36} \mathrm{Ni}\left[\mathrm{Cr}(\mathrm{CN})_{6}\right]_{0.74}\left[\left(\mathrm{H}_{2} \mathrm{O}\right)_{6}\right]_{0.26} \cdot 0.11 \mathrm{H}_{2} \mathrm{O}$, The Journal of Physical Chemistry C 119 (52) (2015) 29138-29147, doi: 10.1021/acs.jpcc.5b07274.

[37] V. Escax, A. Bleuzen, J. P. Itié, P. Munsch, F. Varret, M. Verdaguer, Nature of the Long-Range Structural Changes Induced by the Molecular Photoexcitation and by the Relaxation in the Prussian Blue Analogue $\mathrm{Rb}_{1.8} \mathrm{Co}_{4}\left[\mathrm{Fe}(\mathrm{CN})_{6}\right]_{3.3} \cdot 13 \mathrm{H}_{2} \mathrm{O}$. A Synchrotron X-ray Diffraction Study, The Journal of Physical Chemistry B 107 (20) (2003) 4763-4767, doi: 10.1021/jp0340313.

[38] T. Mallah, S. Thiébaut, M. Verdaguer, P. Veillet, High- $T_{c}$ MolecularBased Magnets: Ferrimagnetic Mixed-Valence Chromium(III)Chromium(II) Cyanides with $\mathrm{T}_{c}$ at 240 and 190 Kelvin, Science 262 (5139) (1993) 1554-1557, doi:10.1126/science.262.5139.1554.

[39] P. A. Quintero, Coordination Polymer Structures: Design and Photomagnetic Properties, Ph.D. thesis, University of Florida, URL http://ufdc.ufl.edu/UFE0049004/00001, 2015.

[40] C. R. Gros, M. K. Peprah, A. C. Felts, T. V. Brinzari, O. N. Risset, J. M. Cain, C. F. Ferreira, M. W. Meisel, D. R. Talham, Synergistic photomagnetic effects in coordination polymer heterostructure particles of Hofmann-like $\mathrm{Fe}(4-$ phenylpyridine $)_{2}\left[\mathrm{Ni}(\mathrm{CN})_{4}\right] \cdot 0.5 \mathrm{H}_{2} \mathrm{O}$ and $\mathrm{K}_{0.4} \mathrm{Ni}\left[\mathrm{Cr}(\mathrm{CN})_{6}\right]_{0.8} \cdot n \mathrm{H}_{2} \mathrm{O}$, Dalton Transactions 45 (2016) 16624-16634, doi:10.1039/C6DT02353C, URL http://dx.doi.org/10.1039/C6DT02353C.

[41] C. R. Gros, M. K. Peprah, B. D. Hosterman, T. V. Brinzari, P. A. Quintero, M. Sendova, M. W. Meisel, D. R. Talham, Light-Induced Magnetization Changes in a Coordination Polymer Heterostructure of a Prussian Blue Analogue and a Hofmann-like Fe(II) Spin Crossover Compound, Journal of the American Chemical Society 136 (28) (2014) 9846-9849, doi:10.1021/ja504289p.

[42] A. Pronschinske, Y. Chen, G. F. Lewis, D. A. Shultz, A. Calzolari, M. B. Nardelli, D. B. Dougherty, Modification of Molecular Spin Crossover in Ultrathin Films, Nano Letters 13 (4) (2013) 1429-1434, doi: $10.1021 / \mathrm{nl} 304304 \mathrm{e}$.
[43] S. Beniwal, X. Zhang, S. Mu, A. Naim, P. Rosa, G. Chastanet, J.-F. Létard, J. Liu, G. E. Sterbinsky, D. A. Arena, P. A. Dowben, A. Enders, Surface-induced spin state locking of the $\left[\mathrm{Fe}\left(\mathrm{H}_{2} \mathrm{~B}(\mathrm{pz})_{2}\right)_{2}\right.$ (bipy)] spin crossover complex, Journal of Physics: Condensed Matter 28 (20) (2016) 206002, doi:10.1088/0953-8984/28/20/206002.

[44] K. Bairagi, O. Iasco, A. Bellec, A. Kartsev, D. Li, J. Lagoute, C. Chacon, Y. Girard, S. Rousset, F. Miserque, Y. J. Dappe, A. Smogunov, C. Barreteau, M.-L. Boillot, T. Mallah, V. Repain, Molecular-scale dynamics of light-induced spin cross-over in a two-dimensional layer, Nature Communications 7 (2016) 12212, doi:10.1038/ncomms12212. 
Table of Contents graphic for Peprah et al. submission to Polyhedron for ICMM2016 Special Issue

Externally applied pressure applied to CoFe@CrCr Prussian blue analogue core@shell nanoparticles shifts the magnetic ordering temperatures of the CoFe-PBA core, the $\mathrm{CrCr}$-PBA shell, and the photoCTIST of the CoFe-PBA core. Specifically, the magnetic ordering temperature of the CrCr-PBA shell shifted to higher temperatures, while the relaxation temperature of the photo-CTIST of the CoFe-PBA core moved to lower temperatures.

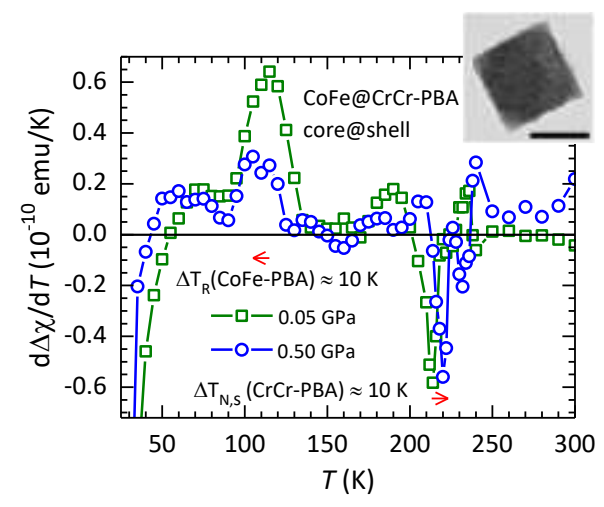

